Article

\title{
Dualistic Hessian Structures Among the Thermodynamic Potentials in the $\kappa$-Thermostatistics
}

\author{
Tatsuaki Wada $^{1, *}$, Hiroshi Matsuzoe ${ }^{2}$ and Antonio M. Scarfone ${ }^{3}$ \\ ${ }^{1}$ Department of Electrical and Electronic Engineering, Ibaraki University, Nakanarusawacho, \\ Hitachi 316-8511, Japan \\ ${ }^{2}$ Department of Computer Science and Engineering, Graduate School of Engineering, Nagoya Institute \\ of Technology, Gokisocho, Nagoya 466-8555, Japan; E-Mail: matsuzoe@ nitech.ac.jp \\ ${ }^{3}$ Istituto dei Sistemi Complessi (ISC-CNR) c/o Politecnico di Torino, Corso Duca degli Abruzzi 24, \\ Torino I-10129, Italy; E-Mail: antonio.scarfone@ polito.it \\ * Author to whom correspondence should be addressed; E-Mail: tatsuaki.wada.to@vc.ibaraki.ac.jp; \\ Tel.: +81-294-38-5110; Fax: +81-294-38-5275.
}

Academic Editor: George Ruppeiner

Received: 28 July 2015 / Accepted: 15 October 2015 / Published: 22 October 2015

\begin{abstract}
We explore the information geometric structures among the thermodynamic potentials in the $\kappa$-thermostatistics, which is a generalized thermostatistics based on the $\kappa$-deformed entropy. We show that there exists two different kinds of dualistic Hessian structures: one is associated with the $\kappa$-escort expectations and the other with the standard expectations. The associated $\kappa$-generalized metrics are derived and related to the $\kappa$-generalized fluctuation-response relations among the thermodynamic potentials in the $\kappa$-thermostatistics.
\end{abstract}

Keywords: $\quad \kappa$-entropy; $\kappa$-exponential; $\kappa$-logarithm; information geometry; Hessian geometry; dually-flat; thermodynamic geometry

\section{Introduction}

The geometric approaches to thermodynamics and statistical mechanics have been developed since the early works of Gibbs [1] and Carathéodory [2]. Ruppeiner [3] and Weinhold [4,5] independently introduced the Riemannian metrics, which are constructed from thermodynamic potentials (entropy or 
internal energy). The thermodynamic fluctuations around the equilibrium states have been studied, and the associated Riemannian curvature has been related to an interaction that characterizes a thermodynamic system. On the other hand, information geometry [6] has been developed mainly in the fields of statistics, and it provides a useful framework for studying the family of probability distributions, mainly the exponential family, by using the geometric tools in affine differential geometry. One of the distinct features in information geometry is a dualistic structure of affine connections, which provides us a very useful tool for many scientific fields, such as information theory, statistics, neural networks, statistical physics, and so on.

Recently, for studying power law distributions, some deformed exponential families [7,8] have attracted attention in various scientific fields. Among the deformed exponential functions, the $\kappa$-deformed exponential function [9] was proposed recently and has been developed in many fields, such as statistical physics [9-12], thermostatistics, financial physics, social science, statistics, information theory and information geometry [13]. Although the physical meaning of the deformed parameter $\kappa$ is not established yet, some theoretical foundations [14] of the $\kappa$-deformed exponential functions have been developed.

The $\kappa$-thermostatistics is a generalization of thermostatistics [15] based on $\kappa$-entropy $S_{\kappa}$, which reduces to the standard Gibbs-Shannon entropy in the limit of $\kappa \rightarrow 0$. Since a deformed exponential probability density function (pdf) naturally induces the escort pdf [8] in general, the $\kappa$-deformed exponential pdf also leads to the $\kappa$-escort pdf. As a result, it is important to take into account the two different kinds of expectations: one is the $\kappa$-escort expectation, and the other is the standard expectation. Accordingly, the $\kappa$-entropy $S_{\kappa}$, which is defined by the standard expectation, naturally induces the $\kappa$-escort entropy, which is expressed as the $\kappa$-escort expectation. While two of the authors (Tatsuaki Wada and Antonio M. Scarfone) studied the information geometric structures [13,16] of the $\kappa$-thermostatistics, the other author (Hiroshi Matsuzoe) showed that a deformed exponential family has two kinds of dualistic Hessian structures [17] in general. We here explore the information geometric structures concerning the $\kappa$-thermostatistics. Remarkably, as shown in this paper, there exist two different kinds of dualistic Hessian structures among the thermodynamic potentials in the $\kappa$-thermostatistics.

In the next section, we begin with a brief review of the geometric approach to thermodynamics and Callen's thermostatistics [15]. Section 3 provides the preliminaries on the Hessian geometry concerning the information geometry based on the exponential family. It also provides the very basics of the $\kappa$-thermostatistics. In Section 4, we explain the dualistic structures of the Hessian geometries in the $\kappa$-thermostatistics. We explore the Hessian structure associated with the Legendre relations for the $\kappa$-entropy. We derive some non-trivial relations, which disappear in the standard limit of $\kappa \rightarrow 0$. The final section is devoted to the conclusions.

\section{Thermodynamics and Thermostatistics}

Consider a thermal equilibrium system characterized by the entropy $S$ as a state function of the internal energy $U$ and volume $V$, i.e., in entropy representation $S=S(U, V)$. We assume that the 
thermal system has a fixed number of particles. As is well known, the first law of thermodynamics is expressed as:

$$
d S=\frac{1}{T} d U+\frac{P}{T} d V
$$

where $T$ and $P$ denote the temperature and the pressure of the thermal system, respectively. They are related by the relations:

$$
\frac{1}{T}=\left(\frac{\partial S}{\partial U}\right)_{V}, \quad \frac{P}{T}=\left(\frac{\partial S}{\partial V}\right)_{U}
$$

Mathematically, these relations are necessary and sufficient conditions, so that the Pfaff equation $d S(U, V)-(1 / T) d U-(P / T) d V=0$ is an exact differential, and consequently, the entropy $S(U, V)$ is a state function, as shown originally by Carathéodory [2]. Planck potential $\Xi$ is given by:

$$
\Xi\left(\frac{1}{T}, \frac{P}{T}\right)=S(U, V)-\frac{1}{T} U-\frac{P}{T} V,
$$

which is nothing but the total Legendre transform of $S(U, V)$.

For the sake of later convenience, instead of the concave function $S(U, V)$, we use the convex function $-S(U, V)$, which is called negentropy, or negative entropy [18]. Introducing the set of the extensive variables $\eta_{i},(i=1,2)$ with $\eta_{1}=U, \eta_{2}=V$, and the set of the intensive variables $\theta^{i},(i=1,2)$ with $\theta^{1}=-1 / T, \theta^{2}=-P / T$, Relations (2) can be compactly expressed as:

$$
\theta^{i}=\partial^{i}(-S), \quad i=1,2
$$

where $\partial^{i}=\partial / \partial \eta_{i}$. The Legendre relation (3) becomes:

$$
\Xi\left(\theta^{1}, \theta^{2}\right)=\sum_{m=1}^{2} \theta^{m} \eta_{m}+S\left(\eta_{1}, \eta_{2}\right)
$$

and the dual relation of (4) is readily obtained from (5) as:

$$
\eta_{i}=\partial_{i} \Xi, \quad i=1,2 .
$$

where $\partial_{i}=\partial / \partial \theta^{i}$.

It is also known that the Maxwell relations in thermodynamics are due to the irrelevance of the order of differentiating a thermodynamic potential (an analytic function) with respect to two variables, For instance, for the negentropy $-S$, the following Maxwell relation:

$$
\frac{\partial}{\partial V}\left(\frac{1}{T}\right)=\frac{\partial}{\partial U}\left(\frac{P}{T}\right)
$$

is equivalent to the relation:

$$
\partial^{2} \partial^{1}(-S)=\partial^{1} \partial^{2}(-S)
$$

The Hessian of the negentropy $(-S)$ can be considered as a symmetric metric tensor of a manifold with the thermodynamic variables $\eta_{i}$ as its coordinates,

$$
\left(g^{\mathrm{R}}\right)^{i j} \equiv \partial^{i} \partial^{j}(-S)
$$


which is equivalent to the Ruppeiner metric [3]. The inverse matrix of $\left(g^{\mathrm{R}}\right)^{i j}$ is given by:

$$
g_{i j}^{\mathrm{R}}=\partial_{i} \partial_{j} \Xi
$$

In Callen's thermostatistics [15], the concept of the equilibrium states in conventional thermal physics is extended to the "equilibrium states", which are characterized as the states that maximize the disorder, or the measure of information. A well-known measure of information is the Gibbs-Shannon entropy $S$, which is expressed as the expectation of $-\ln p(x ; \boldsymbol{\theta})$, i.e.,

$$
S=\mathrm{E}_{p}[-\ln p(x ; \boldsymbol{\theta})] .
$$

Here and hereafter, $\mathrm{E}_{p}[\cdot]$ stands for the standard expectation with respect to a pdf $p(x ; \boldsymbol{\theta})$, which is characterized by the parameter $\boldsymbol{\theta}=\left(\theta^{1}, \theta^{2}, \ldots, \theta^{M}\right)$ with an appropriate degree of freedom $M$. Any extensive thermal quantity is considered as the expectation (or average) of the corresponding microscopic quantity, for instance the internal energy is given by:

$$
U=\int d x p(x ; T, P) E(x),
$$

where $E(x)$ is the microscopic energy of a configuration $x$ and $p(x ; T, P)$ is a pdf depending on the intensive parameters $T$ and $P$. Introducing the notation $f_{i}(x)$ for the microscopic quantity associated with $\eta_{i}$, we can express the extensive thermal quantities as:

$$
\eta_{i}=\int d x p(x ; \boldsymbol{\theta}) f_{i}(x)=\mathrm{E}_{p}\left[f_{i}(x)\right], \quad i=1,2, \ldots, M .
$$

From the Legendre transformation (5) and Equation (13), we see that:

$$
\mathrm{E}_{p}[\ln p(x ; \boldsymbol{\theta})]=-S(\boldsymbol{\eta})=\sum_{m} \theta^{m} \mathrm{E}_{p}\left[f_{m}(x)\right]-\Xi(\boldsymbol{\theta})=\mathrm{E}_{p}\left[\sum_{m} \theta^{m} f_{m}(x)-\Xi(\boldsymbol{\theta})\right] .
$$

We thus obtain that:

$$
\ln p(x ; \boldsymbol{\theta})=\sum_{m} \theta^{m} f_{m}(x)-\Xi(\boldsymbol{\theta}),
$$

and consequently, we see that the $p(x ; \boldsymbol{\theta})$ is an exponential pdf:

$$
p(x ; \boldsymbol{\theta})=\exp \left(\sum_{m} \theta^{m} f_{m}(x)-\Xi(\boldsymbol{\theta})\right) .
$$

The quantity:

$$
\partial_{i} \ell_{\theta}(x) \equiv \partial_{i} \ln p(x ; \boldsymbol{\theta}), \quad i=1,2, \ldots, M,
$$

is called the score function in statistics, and it has zero expectation, i.e.,

$$
\mathrm{E}_{p}\left[\partial_{i} \ell_{\theta}(x)\right] \equiv \int d x p(x ; \boldsymbol{\theta}) \partial_{i} \ln p(x ; \boldsymbol{\theta})=\int d x \partial_{i} p(x ; \boldsymbol{\theta})=\partial_{i} \mathrm{E}_{p}[1]=0,
$$

which is due to the normalization $\mathrm{E}_{p}[1]=1$ of any pdf $p(x ; \boldsymbol{\theta})$. From this, we readily confirm Relation (6) as:

$$
0=\mathrm{E}_{p}\left[\partial_{i} \ell_{\theta}(x)\right]=\mathrm{E}_{p}\left[f_{i}(x)\right]-\partial_{i} \Xi(\boldsymbol{\theta})=\eta_{i}-\partial_{i} \Xi(\boldsymbol{\theta}) .
$$


Let us introduce Fisher's information matrix $g_{i j}^{\mathrm{F}}$ defined by:

$$
g_{i j}^{\mathrm{F}}(\boldsymbol{\theta}) \equiv \mathrm{E}_{p}\left[\partial_{i} \ell_{\theta}(x) \partial_{j} \ell_{\theta}(x)\right] . \quad i, j=1,2, \ldots, M
$$

Differentiating both sides of Equation (18) with respect to $\theta$, we obtain:

$$
\int d x \partial_{i} p(x ; \boldsymbol{\theta}) \partial_{j} \ell_{\theta}(x)=-\int d x p(x ; \boldsymbol{\theta}) \partial_{i} \partial_{j} \ell_{\theta}(x) .
$$

Using this relation, the Fisher metric $g^{\mathrm{F}}$ can be written equivalently in other different expressions:

$$
\begin{aligned}
g_{i j}^{\mathrm{F}} & =\int d x \partial_{i} p(x ; \boldsymbol{\theta}) \partial_{j} \ell_{\theta}(x) \\
& =-\int d x p(x ; \boldsymbol{\theta}) \partial_{i} \partial_{j} \ell_{\theta}(x) \\
& =\int d x \frac{1}{p(x ; \boldsymbol{\theta})} \partial_{i} p(x ; \boldsymbol{\theta}) \partial_{j} p(x ; \boldsymbol{\theta}) .
\end{aligned}
$$

In particular, substituting Equation (15) into (23), we readily confirm that:

$$
g_{i j}^{\mathrm{F}}=\partial_{i} \partial_{j} \Xi(\boldsymbol{\theta})=g_{i j}^{\mathrm{R}},
$$

that is this Fisher metric for the exponential pdf (16) is a Hessian matrix and coincides with the inverse matrix of Ruppeiner metric $\left(g^{\mathrm{R}}\right)^{i j}$.

\section{Preliminaries}

In information geometry [6], a pair of dually-flat affine connections plays an essential role in the geometrical methods of statistical inference. A well-known dually-flat space is the statistical manifold of the exponential family, which can be naturally considered as a Hessian manifold.

\subsection{Hessian Geometry}

We here briefly review the basics of the Hessian manifold. For more details, please see [17]. Let $\mathcal{M}$ be a manifold, $h$ be a positive definite metric and $(\mathcal{M}, h)$ be a Riemannian manifold. For an affine connection $\nabla$, we can define the dual connection $\nabla^{\star}$ of $\nabla$ associated with $h$ by:

$$
X h(Y, Z)=h\left(\nabla_{X} Y, Z\right)+h\left(Y, \nabla_{X}^{\star} Z\right)
$$

where $X, Y$ and $Z$ are vector fields on $\mathcal{M}$. The affine connection $\nabla$ is also the dual of $\nabla^{\star}$. We say that $\nabla$ is curvature free if the curvature tensor:

$$
R(X, Y) Z \equiv \nabla_{X} \nabla_{Y} Z-\nabla_{Y} \nabla_{X} Z-\nabla_{[X, Y]} Z
$$

vanishes everywhere on $\mathcal{M}$. Here, $[X, Y] \equiv X Y-Y X$. The torsion tensor $\mathcal{T}$ is defined by:

$$
\mathcal{T}(X, Y) \equiv \nabla_{X} Y-\nabla_{Y} X-[X, Y]
$$

and we say that $\nabla$ is torsion free if $\mathcal{T}$ vanishes everywhere on $\mathcal{M}$. 
An affine connection $\nabla$ is assumed to be torsion free in this study. If an affine connection $\nabla$ is curvature free, we say that the $\nabla$ is flat. In this case, there exists a coordinate system $\left\{\theta^{i}\right\}$ on $\mathcal{M}$ locally, such that the connection coefficients $\left\{\Gamma_{i j}^{k}\right\}$ of $\nabla$ vanish on the coordinate neighborhood. Such a coordinate system $\left\{\theta^{i}\right\}$ is called an affine coordinate system.

For a Riemannian manifold $(\mathcal{M}, h)$ and a flat affine connection $\nabla$ on $\mathcal{M}$, the set $(\nabla, h)$ is called a Hessian structure on $\mathcal{M}$ if there exists, at least locally, a function $\Psi$, such that $h=\nabla d \Psi$. This is expressed, in the coordinate form, as:

$$
h_{i j}\left(\boldsymbol{\theta}_{p}\right)=\partial_{i} \partial_{j} \Psi\left(\boldsymbol{\theta}_{p}\right)
$$

where $\boldsymbol{\theta}_{p}$ is the coordinate of an arbitrary point $p$ on $\mathcal{M}$.

It is known that for a Hessian manifold $(\mathcal{M}, \nabla, h)$ and the dual coordinate systems $\left\{\theta^{i}\right\}$ for $\nabla$ and $\left\{\eta_{i}\right\}$ for $\nabla^{\star}$, there exists a pair of the potential functions $\Psi$ and $\Psi^{\star}$ on $\mathcal{M}$, such that:

$$
\begin{aligned}
\Psi\left(\boldsymbol{\theta}_{p}\right) & +\Psi^{\star}\left(\boldsymbol{\eta}_{p}\right)-\boldsymbol{\theta}_{p} \cdot \boldsymbol{\eta}_{p}=0, \\
\theta^{i} & =\partial^{i} \Psi^{*}(\boldsymbol{\eta}), \quad \eta_{i}=\partial_{i} \Psi(\boldsymbol{\theta}), \\
h_{i j} & =\partial_{i} \partial_{j} \Psi(\boldsymbol{\theta}), \quad h^{i j}=\partial^{i} \partial^{j} \Psi^{\star}(\boldsymbol{\eta}),
\end{aligned}
$$

where the matrix $h_{i j}$ of a Riemannian metric $h(\boldsymbol{\theta})$ is the inverse matrix $h^{i j}$ of $h(\boldsymbol{\eta})$, and vice versa. The potential functions $\Psi(\boldsymbol{\theta})$ and $\Psi^{*}(\boldsymbol{\eta})$ are Legendre dual to each other and are called $\theta$ - and $\eta$-potential functions, respectively. Note also that $\left(\mathcal{M}, \nabla^{\star}, h\right)$ is a Hessian manifold associated with the potential function $\Psi^{\star}$.

For the exponential pdf:

$$
p(x ; \boldsymbol{\theta})=\exp \left(\sum_{m} \theta^{m} f_{m}(x)-\Psi(\boldsymbol{\theta})\right),
$$

it is well known that Fisher metric $g^{\mathrm{F}}$ is expressed as the Hessian of the potential function, e.g.,:

$$
g_{i j}^{\mathrm{F}}(\boldsymbol{\theta})=\partial_{i} \partial_{j} \Psi(\boldsymbol{\theta}) .
$$

From Equation (33), we obtain

$$
\partial_{i} \ell_{\theta}(x)=\partial_{i} \ln p(x ; \boldsymbol{\theta})=f_{i}(x)-\partial_{i} \Psi(\boldsymbol{\theta}) .
$$

Substituting this relation into Definition (20) and using $\eta_{i}=\partial_{i} \Psi(\boldsymbol{\theta})$, we see that:

$$
g_{i j}^{\mathrm{F}}(\boldsymbol{\theta})=\mathrm{E}_{p}\left[\left(f_{i}-\mathrm{E}_{p}\left[f_{i}\right]\right)\left(f_{j}-\mathrm{E}_{p}\left[f_{j}\right]\right)\right], \quad i, j=1,2, \ldots, M,
$$

which is the covariance matrix characterizing the expectation of the fluctuations around the expectations $\mathrm{E}_{p}\left[f_{i}\right]$. Physically, this means that the expectations of the relevant thermodynamic fluctuations characterize the metric $g_{i j}^{\mathrm{F}}$ of the statistical manifold $\mathcal{M}$ for the equilibrium thermodynamics. In addition, since $g_{i j}^{\mathrm{F}}=\partial^{i} \eta_{j}$, each component of $g_{i j}^{\mathrm{F}}$ describes a response function, which is the derivative of an extensive variable $\eta_{j}$ with respect to an intensive variable $\theta^{i}$. As a result, the physical interpretation of Relation (36) is given by the fluctuation-response relations $[15,16]$ for an equilibrium thermal system. 
The canonical divergence function [6] for the two points $p$ and $r$ on $\mathcal{M}$ can be defined by:

$$
D(\boldsymbol{p}, \boldsymbol{r}) \equiv \Psi\left(\boldsymbol{\theta}_{p}\right)+\Psi^{\star}\left(\boldsymbol{\eta}_{r}\right)-\boldsymbol{\theta}_{p} \cdot \boldsymbol{\eta}_{r}
$$

It is well known that for the exponential pdf, the canonical divergence coincides with the Kullback-Leibler (KL) divergence. In addition, for the exponential pdf $p(x ; \boldsymbol{\theta})$ and an arbitrary pdf $r(x)$, we have:

$$
\int d x r(x) \ln p(x ; \boldsymbol{\theta})=\int d x r(x)\left[\sum_{m} \theta^{m} f_{m}(x)-\Psi\left(\boldsymbol{\theta}_{p}\right)\right]=\boldsymbol{\theta}_{p} \cdot \boldsymbol{\eta}_{r}-\Psi\left(\boldsymbol{\theta}_{p}\right) .
$$

Then, it follows that:

$$
\begin{aligned}
D(\boldsymbol{p}, \boldsymbol{r}) & =\Psi^{\star}\left(\boldsymbol{\eta}_{r}\right)-\left(\boldsymbol{\theta}_{p} \cdot \boldsymbol{\eta}_{r}-\Psi\left(\boldsymbol{\theta}_{p}\right)\right)=\int d x r(x)[\ln r(x)-\ln p(x ; \boldsymbol{\theta})] \\
& =\int d x r(x) \ln \left(\frac{r(x)}{p(x ; \boldsymbol{\theta})}\right) .
\end{aligned}
$$

The dual affine connections $\nabla^{(e)}$ and $\nabla^{(m)}$ are induced from the Fisher metric. The Christoffel symbol $\Gamma_{i j, k}^{(e)}$ of the first kind for the e-affine connection $\nabla^{(e)}$ and that $\Gamma_{i j, k}^{(m)}$ for the m-affine connection $\nabla^{(m)}$ are defined so that the next relation holds: [6]:

$$
\partial_{i} g_{j k}^{\mathrm{F}}=\Gamma_{i j, k}^{(e)}+\Gamma_{i j, k}^{(m)}
$$

More specifically, they are explicitly given by:

$$
\begin{aligned}
\Gamma_{i j, k}^{(e)} & \equiv \int d x \partial_{k} p(x ; \boldsymbol{\theta}) \partial_{i} \partial_{j} \ell_{\theta}(x)=\mathrm{E}_{p}\left[\partial_{k} \ell_{\theta} \partial_{i} \partial_{j} \ell_{\theta}(x)\right] \\
\Gamma_{i j, k}^{(m)} & \equiv \int d x \partial_{i} \partial_{j} p(x ; \boldsymbol{\theta}) \partial_{k} \ell_{\theta}(x)=\mathrm{E}_{p}\left[\frac{1}{p(x ; \boldsymbol{\theta})} \partial_{i} \partial_{j} p(x ; \boldsymbol{\theta}) \partial_{k} \ell_{\theta}(x)\right],
\end{aligned}
$$

respectively.

\section{2. $\kappa$-Thermostatistics}

The $\kappa$-thermostatistics is a generalized thermostatistics [8] based on the $\kappa$-entropy $S_{\kappa}$ given by:

$$
S_{\kappa} \equiv-\int d x p(x) \ln _{\kappa} p(x)=\mathrm{E}_{p}\left[-\ln _{\kappa} p\right]
$$

which mimics the Gibbs-Shannon entropy (11) by replacing the standard logarithm with the $\kappa$-logarithm. Here, the $\kappa$-logarithm $\ln _{\kappa} x[10,11]$ is a deformed function of the standard logarithm $\ln x$ with a real parameter $\kappa \in(-1,1)$.

$$
\ln _{\kappa} x \equiv \frac{x^{\kappa}-x^{-\kappa}}{2 \kappa}=\frac{1}{\kappa} \sinh (\kappa \ln x),
$$

for $x>0$. Its inverse function is given by:

$$
\exp _{\kappa}(x) \equiv\left(\kappa x+\sqrt{1+\kappa^{2} x^{2}}\right)^{\frac{1}{\kappa}}=\exp \left[\frac{1}{\kappa} \operatorname{arcsinh}(\kappa x)\right]
$$


In the $\kappa \rightarrow 0$ limit, the $\kappa$-exponential and the $\kappa$-logarithm reduce to the standard exponential $\exp (x)$ and logarithm $\ln x$, respectively.

We introduce another $\kappa$-deformed function:

$$
u_{\kappa}(x) \equiv \frac{x^{\kappa}+x^{-\kappa}}{2}=\cosh (\kappa \ln x)
$$

which is, in a sense, the conjugate (or co-function) of $\ln _{\kappa} x$, as $\cos x$ is the co-function of $\sin x$. In the $\kappa \rightarrow 0$ limit, this $\kappa$-deformed function reduces to the unit constant function $u_{0}(x)=1$. Similar to the $\kappa$-entropy $S_{\kappa}$ being defined as the expectation of $-\ln _{\kappa} p(x)$, we introduce the following function:

$$
\mathcal{I}_{\kappa} \equiv \int d x p(x) u_{\kappa}(p(x))=\mathrm{E}_{p}\left[u_{\kappa}(p)\right]
$$

as the expectation of $u_{\kappa}(p(x))$. The $\kappa$-dependent constant $\alpha$ and $\lambda$ are introduced so that they satisfy the following equations [13]:

$$
\begin{aligned}
\frac{d}{d x}\left(x \ln _{\kappa} x\right) & =\lambda \ln _{\kappa}\left(\frac{x}{\alpha}\right)=\ln _{\kappa} x+u_{\kappa}(x), \\
\frac{d}{d x}\left(x u_{\kappa}(x)\right) & =\lambda u_{\kappa}\left(\frac{x}{\alpha}\right)=u_{\kappa}(x)+\kappa^{2} \ln _{\kappa} x,
\end{aligned}
$$

for any $x>0$. These equations for the $\alpha$ and $\lambda$ can be derived [12] from the MaxEnt principle for the $\kappa$-entropy $S_{\kappa}$, and the solutions are expressed as:

$$
\alpha=\left(\frac{1-\kappa}{1+\kappa}\right)^{\frac{1}{2 \kappa}}, \quad \lambda=\sqrt{1-\kappa^{2}},
$$

which are related to each other according to:

$$
\alpha=\exp _{\kappa}\left(-\frac{1}{\lambda}\right)
$$

Note that in the standard limit of $\kappa=0$, the parameter $\alpha$ reduces to $\exp (-1)$, and Relation (48) reduces to the well-known form:

$$
\frac{d}{d x}(x \ln x)=\ln x+1
$$

In other words, Relation (48) is a $\kappa$-generalization of the standard Relation (52). In addition, Relation (49) is non-trivial unless $\kappa=0$, since it reduces to the trivial identity $1=1$ when $\kappa=0$.

\section{Dual Structures of the Hessian Geometries in the $\kappa$-Thermostatistics}

In [16,19], we have derived the Legendre relations from the $\kappa$-deformed exponential pdf (see Equation (59)), which maximizes the $\kappa$-entropy $S_{\kappa}$ under the constraints:

$$
\eta_{m}=\mathrm{E}_{p}\left[f_{m}\right], \quad m=1,2, \cdots, M,
$$

and the normalization of the pdf. Here, we follow the reverse order of the above derivation, i.e., starting from the Legendre relations, we derive the $\kappa$-deformed exponential pdf. We assume the Legendre relations for the $\theta$ - and $\eta$ - potential functions $\Psi_{\kappa}(\boldsymbol{\theta})$ and $\Psi_{\kappa}^{\star}(\boldsymbol{\eta})$ :

$$
\begin{aligned}
\Psi_{\kappa}(\boldsymbol{\theta}) & =\boldsymbol{\theta} \cdot \boldsymbol{\eta}-\Psi_{\kappa}^{\star}(\boldsymbol{\eta}), \\
\theta^{i} & =\partial^{i} \Psi_{\kappa}^{\star}(\boldsymbol{\eta}), \quad \eta_{i}=\partial_{i} \Psi_{\kappa}(\boldsymbol{\theta})
\end{aligned}
$$


with $\eta_{m}=\mathrm{E}_{p}\left[f_{m}\right]$ and recognizing the potential functions as:

$$
\Psi_{\kappa}^{\star}(\boldsymbol{\eta})=-S_{\kappa}(\boldsymbol{\eta}), \quad \Psi_{\kappa}(\boldsymbol{\theta})=\mathcal{I}_{\kappa}(\boldsymbol{\theta})+\gamma(\boldsymbol{\theta}),
$$

Then, we can derive the $\kappa$-deformed exponential pdf as follows. Using the useful Identity (48), we see that:

$$
\begin{aligned}
\mathrm{E}_{p}\left[\lambda \ln _{\kappa}\left(\frac{p(x ; \boldsymbol{\theta})}{\alpha}\right)\right] & =\mathrm{E}_{p}\left[\ln _{\kappa}(p(x ; \boldsymbol{\theta}))\right]+\mathrm{E}_{p}\left[u_{\kappa}(p(x ; \boldsymbol{\theta})]=-S_{\kappa}(\boldsymbol{\eta})+\mathcal{I}_{\kappa}(\boldsymbol{\theta})\right. \\
& =\Psi_{\kappa}^{\star}(\boldsymbol{\eta})+\Psi_{\kappa}(\boldsymbol{\theta})-\gamma(\boldsymbol{\theta})=\sum_{m=1}^{M} \theta^{m} \mathrm{E}_{p}\left[f_{m}\right]-\gamma(\boldsymbol{\theta}) \\
& =\mathrm{E}_{p}\left[\sum_{m=1}^{M} \theta^{m} f_{m}(x)-\gamma(\boldsymbol{\theta})\right] .
\end{aligned}
$$

Comparing the both sides, we obtain that:

$$
\lambda \ln _{\kappa}\left(\frac{p(x ; \boldsymbol{\theta})}{\alpha}\right)=\sum_{m=1}^{M} \theta^{m} f_{m}(x)-\gamma(\boldsymbol{\theta}),
$$

from which the $\kappa$-deformed exponential pdf:

$$
p(x ; \boldsymbol{\theta})=\alpha \exp _{\kappa}\left[\frac{1}{\lambda}\left(\sum_{m=1}^{M} \theta^{m} f_{m}(x)-\gamma(\boldsymbol{\theta})\right)\right],
$$

is derived. Note that unlike in the standard case in which a relevant pdf is exponential, the parameter $\theta^{i}$ is not necessarily intensive and the variable $\eta_{i}$ is not necessarily extensive in general. In order to avoid misleading, we call $\theta^{i}$ an external parameter, which characterize a state of the thermal systems described by the $\kappa$-deformed exponential pdf (59), and we call $\eta_{i}$ an expectation variable.

For a deformed exponential pdf, it is known [8] that the so-called escort pdf is naturally induced. We thus introduce the $\kappa$-escort pdf $P(x ; \boldsymbol{\theta})[13]$ with respect to $p(x ; \boldsymbol{\theta})$ by:

$$
P(x ; \boldsymbol{\theta}) \equiv \frac{1}{\mathcal{U}_{\kappa}} \frac{p(x ; \boldsymbol{\theta})}{\lambda u_{\kappa}\left(\frac{p(x ; \boldsymbol{\theta})}{\alpha}\right)},
$$

where $\mathcal{U}_{\kappa}$ is the normalization factor:

$$
\mathcal{U}_{\kappa} \equiv \int d x \frac{p(x ; \boldsymbol{\theta})}{\lambda u_{\kappa}\left(\frac{p(x ; \boldsymbol{\theta})}{\alpha}\right)},
$$

and the corresponding $\kappa$-escort expectation $\mathbb{E}_{P}[A]$ of a function $A(x)$ is defined by:

$$
\mathbb{E}_{P}[A] \equiv \int d x P(x ; \boldsymbol{\theta}) A(x) .
$$

Having described the basics concerning $\kappa$-thermostatistics, we now consider its Hessian geometry. The next theorem relates a generalized score function $s(x)$ to the generalized pdf $\mathcal{P}(x)$ for which the expectation of $s(x)$ becomes zero. This zero-expectation of a generalized score function $s(x)$ is an important and useful property, which correctly leads to the Legendre relations between an expectation value $\eta_{i}$ and the relevant thermodynamic potential, as shown in (19) for the standard score function (17). 
Theorem 1. For the general score function in the form:

$$
s(x)=\partial_{i} \Lambda(p(x))
$$

with a given smooth differentiable functional $\Lambda(p)$ of a pdf $p(x)$, the expectation of $s(x)$ with respect to the $\mathcal{P}(x)$ becomes zero if we choose:

$$
\left.\mathcal{P}(x) \propto \frac{\partial \Lambda^{-1}(t)}{\partial t}\right|_{t=\Lambda(p(x))}
$$

where $\Lambda^{-1}(t)$ denotes the inverse function of $\Lambda(t)$.

Proof. For any pdf $p(x), \int d x \partial_{i} p(x)=0$ because of the normalization $\int d x p(x)=1$. Then:

$$
\int d x \mathcal{P}(x) s_{\theta}(x)=0
$$

if the condition:

$$
\mathcal{P}(x) \partial_{i} \Lambda(p(x))=\mathcal{P}(x) \frac{\partial \Lambda(p)}{\partial p} \partial_{i} p(x) \propto \partial_{i} p(x)
$$

is satisfied. This means that:

$$
\mathcal{P}(x) \propto \frac{1}{\partial \Lambda(p) / \partial p}=\left.\frac{\partial \Lambda^{-1}(t)}{\partial t}\right|_{t=\Lambda(p(x))}
$$

Needless to say, an appropriate proportional factor is needed for the normalization of $\mathcal{P}(x)$.

A well-known example is given by $\Lambda(p)=\ell_{\theta}(x)=\ln p(x ; \boldsymbol{\theta})$, i.e., the score function of (17). In this case, $\mathcal{P}=p(x ; \boldsymbol{\theta})$, and Relation (18) is satisfied.

Now, let us introduce the $\kappa$-generalization $\ell_{\theta}^{(\kappa)}$ of $\ell_{\theta}(x)$ :

$$
\ell_{\theta}^{(\kappa)} \equiv \lambda \ln _{\kappa}\left(\frac{p(x ; \boldsymbol{\theta})}{\alpha}\right)
$$

which reduces to $\ell_{\theta}(x)+1$ in the limit of $\kappa \rightarrow 0$. The inverse function of $\ell_{\theta}^{(\kappa)}$ is:

$$
\left(\ell_{\theta}^{(\kappa)}\right)^{(-1)}(t)=\alpha \exp _{\kappa}\left(\frac{t}{\lambda}\right)
$$

and by using the relation:

$$
\frac{d}{d x} \exp _{\kappa}(x)=\frac{\exp _{\kappa}(x)}{u_{\kappa}\left(\exp _{\kappa}(x)\right)}
$$

we have:

$$
\left.\frac{\partial\left(\ell_{\theta}^{(\kappa)}\right)^{(-1)}(t)}{\partial t}\right|_{t=\ell_{\theta}^{(\kappa)}}=\frac{p(x ; \boldsymbol{\theta})}{\lambda u_{\kappa}\left(\frac{p(x ; \boldsymbol{\theta})}{\alpha}\right)} \propto P(x),
$$

which is the $\kappa$-escort pdf (60).

From Theorem 1, we see that the $\kappa$-score function $\partial_{i} \ell_{\theta}^{(\kappa)}$ has zero $\kappa$-escort expectation:

$$
\mathbb{E}_{P}\left[\partial_{i} \ell_{\theta}^{(\kappa)}\right]=0
$$


In addition, for the $\kappa$-exponential pdf (59), the $\kappa$-score function $\partial_{i} \ell_{\theta}^{(\kappa)}$ becomes:

$$
\partial_{i} \ell_{\theta}^{(\kappa)}=f_{i}(x)-\partial_{i} \gamma(\boldsymbol{\theta})
$$

and consequently, we have:

$$
\eta_{i}^{\mathrm{esc}} \equiv \mathbb{E}_{P}\left[f_{i}\right]=\partial_{i} \gamma(\boldsymbol{\theta})
$$

where $\eta_{i}^{\text {esc }}$ is the dual coordinate of $\theta^{i}$ associated with the $\kappa$-escort expectations $\left\{\mathbb{E}_{P}\left[f_{i}\right]\right\}$. Note also that the function $\gamma(\boldsymbol{\theta})$ is the $\theta$-potential function associated with the $\kappa$-escort expectations $\left\{\mathbb{E}_{P}\left[f_{i}\right]\right\}$. In this way, we have two different kinds of dual coordinates $\eta_{i}$ and $\eta_{i}^{\text {esc }}$ for the same $\theta^{i}$ coordinate. We also see that:

$$
\begin{aligned}
\partial^{i}\left(\mathbb{E}_{P}\left[\ell_{\theta}^{\kappa}\right]-1\right) & =\partial^{i} \mathbb{E}_{P}\left[\lambda \ln _{\kappa}(p(x ; \theta) / \alpha)\right]=\partial^{i} \mathbb{E}_{P}\left[\sum_{m} \theta^{m} f_{m}(x)-\gamma\right]=\partial^{i}\left(\sum_{m} \theta^{m} \mathbb{E}_{P}\left[f_{m}\right]-\gamma\right) \\
& =\partial^{i}\left(\sum_{m} \theta^{m} \eta_{m}^{e s c}-\gamma\right)=\theta^{i}+\sum_{m}\left(\partial^{i} \theta^{m}\right) \eta_{m}^{e s c}-\partial^{i} \gamma=\theta^{i}
\end{aligned}
$$

where in the last step, we used:

$$
\partial^{i} \gamma=\sum_{m}\left(\partial^{i} \theta^{m}\right) \partial_{m} \gamma=\sum_{m}\left(\partial^{i} \theta^{m}\right) \eta_{m}^{e s c}
$$

The Legendre relations concerning $\boldsymbol{\eta}^{\text {esc }}$ are summarized as follows:

$$
\begin{aligned}
\Psi_{\kappa}^{\mathrm{esc}}(\boldsymbol{\theta}) & =\gamma(\boldsymbol{\theta})+1, & \left(\Psi_{\kappa}^{\mathrm{esc}}\right)^{\star}\left(\boldsymbol{\eta}^{\mathrm{esc}}\right)=\mathbb{E}_{P}\left[\ell_{\theta}^{(\kappa)}\right]-1, \\
\eta_{i}^{\mathrm{esc}} & =\mathbb{E}_{P}\left[f_{i}\right]=\partial_{i} \gamma(\boldsymbol{\theta}), & \theta_{i}=\frac{\partial}{\partial \eta_{i}^{\mathrm{esc}}}\left(\Psi_{\kappa}^{\mathrm{esc}}\right)^{\star}\left(\boldsymbol{\eta}^{\mathrm{esc}}\right), \\
\left(\Psi_{\kappa}^{\mathrm{esc}}\right)^{\star}\left(\boldsymbol{\eta}^{\mathrm{esc}}\right) & =\boldsymbol{\theta} \cdot \boldsymbol{\eta}^{\mathrm{esc}}-\Psi_{\kappa}^{\mathrm{esc}}(\boldsymbol{\theta}) . &
\end{aligned}
$$

Of course, in the $\kappa \rightarrow 0$ limit, they reduce to the standard relations, respectively. Note that $\left(\Psi_{\kappa}^{\mathrm{esc}}\right)^{\star}\left(\boldsymbol{\eta}^{\mathrm{esc}}\right)$ is the $\kappa$-escort entropy, which is given by the $\kappa$-escort expectation.

Due to Equation (72), the $\kappa$-score function $\partial_{i} \ell_{\theta}^{(\kappa)}$ has non-zero expectation:

$$
\mathrm{E}_{p}\left[\partial_{i} \ell_{\theta}^{(\kappa)}\right] \neq 0
$$

in other words, it is biased. We then introduce the bias correction term $\mathrm{E}_{p}\left[\partial_{i} \ell_{\theta}^{(\kappa)}\right]$ as:

$$
\partial_{i} \ell_{\theta}^{(\kappa)}-\mathrm{E}_{p}\left[\partial_{i} \ell_{\theta}^{(\kappa)}\right]
$$

However, as we will show below, it is remarkable that the bias correction term is expressed in terms of the function $\mathcal{I}_{\kappa}$ in Equation (47).

Theorem 2. For the $\kappa$-score function $\partial_{i} \ell_{\theta}^{(\kappa)}$ with the $\kappa$-generalized representation $\ell_{\theta}^{(\kappa)}$ of Equation (68), the bias correction term is:

$$
\mathrm{E}_{p}\left[\partial_{i} \ell_{\theta}^{(\kappa)}\right]=\partial_{i} \mathcal{I}_{\kappa}(\boldsymbol{\theta})
$$

where $\mathcal{I}_{\kappa}(\boldsymbol{\theta})$ is the expectation of $u_{\kappa}(p(x ; \boldsymbol{\theta}))$. 
Proof. The direct calculation shows that:

$$
\begin{aligned}
\mathrm{E}_{p}\left[\partial_{i} \ell_{\theta}^{(\kappa)}\right] & =\int d x p(x ; \boldsymbol{\theta}) \partial_{i} \lambda \ln _{\kappa}\left(\frac{p(x ; \boldsymbol{\theta})}{\alpha}\right)=\int d x p(x ; \boldsymbol{\theta}) \frac{\lambda u_{\kappa}\left(\frac{p(x ; \boldsymbol{\theta})}{\alpha}\right)}{p(x ; \boldsymbol{\theta})} \partial_{i} p(x ; \boldsymbol{\theta}) \\
& =\int d x \lambda u_{\kappa}\left(\frac{p(x ; \boldsymbol{\theta})}{\alpha}\right) \partial_{i} p(x ; \boldsymbol{\theta})=\partial_{i} \int d x p(x ; \boldsymbol{\theta}) u_{\kappa}(p(x ; \boldsymbol{\theta}))=\partial_{i} \mathcal{I}_{\kappa}(\boldsymbol{\theta}),
\end{aligned}
$$

where we used the useful identities (48) and (49).

We hence introduce the modified $\kappa$-representation:

$$
\tilde{\ell}_{\theta}^{(\kappa)} \equiv \ell_{\theta}^{(\kappa)}-\mathcal{I}_{\kappa}(\boldsymbol{\theta})
$$

and the modified $\kappa$-score function given by:

$$
\partial_{i}\left(\ell_{\theta}^{(\kappa)}-\mathcal{I}_{\kappa}(\boldsymbol{\theta})\right)=\partial_{i} \tilde{\ell}_{\theta}^{(\kappa)}
$$

which of course, has zero expectation:

$$
\mathrm{E}_{p}\left[\partial_{i} \tilde{\ell}_{\theta}^{(\kappa)}\right]=0
$$

by construction. Note that the modified $\kappa$-representation $\tilde{\ell}_{\theta}^{(\kappa)}$ reduces to the standard one $\ell_{\theta}(x)$ in the limit of $\kappa \rightarrow 0$, because of $\lim _{\kappa \rightarrow 0} \mathcal{I}_{\kappa}=1$.

With the help of Equation (55), we can derive the following relations:

$$
\begin{aligned}
\tilde{\ell}_{\theta}^{(\kappa)} & =\sum_{m=1}^{M} \theta^{m} f_{m}(x)-\gamma-\mathcal{I}_{\kappa}=\sum_{m=1}^{M} \theta^{m} f_{m}(x)-\Psi_{\kappa}(\boldsymbol{\theta}), \\
\partial_{i} \tilde{\ell}_{\theta}^{(\kappa)} & =f_{i}(x)-\partial_{i} \Psi_{\kappa}(\boldsymbol{\theta}), \\
\partial_{i} \partial_{j} \tilde{\ell}_{\theta}^{(\kappa)} & =-\partial_{i} \partial_{j} \Psi_{\kappa}(\boldsymbol{\theta}),
\end{aligned}
$$

and from Equations (86) and (88), we confirm Relation (55) as:

$$
0=\mathrm{E}_{p}\left[\partial_{i} \tilde{\ell}_{\theta}^{(\kappa)}\right]=\mathrm{E}_{p}\left[f_{i}\right]-\partial_{i} \Psi_{\kappa}(\boldsymbol{\theta})=\eta_{i}-\partial_{i} \Psi_{\kappa}(\boldsymbol{\theta}) .
$$

Now, taking the expectation of the both sides of Equation (73) and using (74) and (82), we obtain the important relation:

$$
\partial_{i} \mathcal{I}_{\kappa}(\boldsymbol{\theta})=\mathrm{E}_{p}\left[\partial_{i} \ell_{\theta}^{(\kappa)}\right]=\mathrm{E}_{p}\left[f_{i}(x)\right]-\partial_{i} \gamma(\boldsymbol{\theta})=\mathrm{E}_{p}\left[f_{i}(x)\right]-\mathbb{E}_{P}\left[f_{i}(x)\right]
$$

Thus, the $\theta$-derivatives of the function $\mathcal{I}_{\kappa}(\boldsymbol{\theta})$ characterizes the difference between the standard and $\kappa$-escort expectations. This sheds light for understanding a physical role of the $\mathcal{I}_{\kappa}$ function. Note that since $\lim _{\kappa \rightarrow 0} \mathcal{I}_{\kappa}(\boldsymbol{\theta})=1$, the difference of the both expectations disappears in the standard limit of $\kappa=0$.

We now consider the $\kappa$-generalized metric, which is the Hessian matrix of the $\kappa$-deformed $\theta$-potential function:

$$
g_{i j}^{(\kappa)} \equiv \partial_{i} \partial_{j} \Psi_{\kappa}(\boldsymbol{\theta})
$$


Accounting for Relation (55), we can rewrite Equation (92) as:

$$
g_{i j}^{(\kappa)}=\partial_{i} \eta_{j}=\partial_{i} \mathrm{E}_{p}\left[f_{j}\right]=\int d x \partial_{i} p(x ; \boldsymbol{\theta}) f(x),
$$

and taking into account the relation:

$$
\partial_{i} p(x ; \boldsymbol{\theta})=\frac{p(x ; \boldsymbol{\theta})}{\lambda u_{\kappa}\left(\frac{p(x ; \boldsymbol{\theta})}{\alpha}\right)}\left(f_{i}(x)-\partial_{i} \gamma\right)=\mathcal{U}_{\kappa} P(x ; \boldsymbol{\theta})\left(f_{i}(x)-\partial_{i} \gamma\right),
$$

we obtain:

$$
\begin{aligned}
g_{i j}^{(\kappa)} & =\partial_{i} \mathrm{E}_{p}\left[f_{j}\right]=\int d x \partial_{i} p(x ; \boldsymbol{\theta}) f_{j}(x)=\mathcal{U}_{\kappa} \int d x P(x ; \boldsymbol{\theta})\left(f_{i}(x)-\partial_{i} \gamma\right) f_{j}(x) \\
& =\mathcal{U}_{\kappa} \int d x P(x ; \boldsymbol{\theta})\left(f_{i}(x)-\mathbb{E}_{P}\left[f_{i}\right]\right) f_{j}(x)=\mathcal{U}_{\kappa} \mathbb{E}_{P}\left[\left(f_{i}(x)-\mathbb{E}_{P}\left[f_{i}\right]\right) f_{j}(x)\right], \\
& =\mathcal{U}_{\kappa} \mathbb{E}_{P}\left[\left(f_{i}-\mathbb{E}_{P}\left[f_{i}\right]\right)\left(f_{j}-\mathbb{E}_{P}\left[f_{j}\right]\right)\right],
\end{aligned}
$$

which states that the response function $\partial_{i} \mathrm{E}_{p}\left[f_{j}\right]$ associated with the expectation $\mathrm{E}_{p}\left[f_{j}\right]$ is related to the fluctuation associated with the $\kappa$-escort expectation. This is a $\kappa$-generalization of the standard fluctuation-response relation, as pointed out firstly by Naudts [8]. In the previous work [16], we applied the result (95) to a $\kappa$-generalization of the grand-canonical ensemble. We emphasized that the response functions for the standard expectations are related to the $\kappa$-escort expectations of the fluctuations around the $\kappa$-escort expectations.

Similarly, we next consider the $\kappa$-generalized escort metric, which is the Hessian matrix of the $\kappa$-escort $\theta$-potential function $\Psi_{\kappa}^{\mathrm{esc}}(\boldsymbol{\theta})$ :

$$
g_{i j}^{(\kappa-\mathrm{esc})} \equiv \partial_{i} \partial_{j} \Psi_{\kappa}^{\mathrm{esc}}(\boldsymbol{\theta})=\partial_{i} \eta_{j}^{\mathrm{esc}}=\partial_{i} \mathbb{E}_{P}\left[f_{j}\right]=\int d x \partial_{i} P(x ; \boldsymbol{\theta}) f(x) .
$$

Taking the derivative of the normalization factor $\mathcal{U}_{\kappa}$ of (61) with respect to $\theta^{i}$, and using Relations (48), (49) and (94), we obtain:

$$
\partial_{i} \mathcal{U}_{\kappa}=\int d x \frac{p(x ; \boldsymbol{\theta}) u_{\kappa}(p(x ; \boldsymbol{\theta}))}{\lambda\left(u_{\kappa}\left(\frac{p(x ; \boldsymbol{\theta})}{\alpha}\right)\right)^{3}}\left(f_{i}(x)-\partial_{i} \gamma(\boldsymbol{\theta})\right) .
$$

Similar to Relation (95), which relates the metric $g^{(\kappa)}$ to the thermodynamic fluctuations associated with the $\kappa$-escort expectation, we can relate the metric $g^{(\kappa-\mathrm{esc})}$ to a certain kind of thermodynamic fluctuation. To this end, based on Relation (97), we introduce the double-escort pdf $\tilde{P}(x ; \boldsymbol{\theta})$ defined by:

$$
\tilde{P}(x ; \boldsymbol{\theta}) \equiv \frac{1}{\tilde{\mathcal{U}}_{\kappa}} \frac{p(x ; \boldsymbol{\theta}) u_{\kappa}(p(x ; \boldsymbol{\theta}))}{\lambda\left(u_{\kappa}\left(\frac{p(x ; \boldsymbol{\theta})}{\alpha}\right)\right)^{3}},
$$

which is the escort pdf of the $\kappa$-escort pdf (60), and $\tilde{\mathcal{U}}_{\kappa}$ denotes the normalization factor:

$$
\tilde{\mathcal{U}}_{\kappa} \equiv \int d x \frac{p(x ; \boldsymbol{\theta}) u_{\kappa}(p(x ; \boldsymbol{\theta}))}{\lambda\left(u_{\kappa}\left(\frac{p(x ; \boldsymbol{\theta})}{\alpha}\right)\right)^{3}},
$$

The double-escort $\kappa$-expectation $\tilde{\mathbb{E}}_{\tilde{P}}[A]$ of a function $A(x)$ is defined by:

$$
\tilde{\mathbb{E}}_{\tilde{P}}[A] \equiv \int d x \tilde{P}(x ; \boldsymbol{\theta}) A(x) .
$$


By using the double-escort $\kappa$-expectation, Relation (97) is expressed as:

$$
\partial_{i} \mathcal{U}_{\kappa}=\tilde{\mathcal{U}}_{\kappa}\left(\tilde{\mathbb{E}}_{\tilde{P}}\left[f_{i}\right]-\mathbb{E}_{P}\left[f_{i}\right]\right),
$$

which is similar to Relation (91), and we see that the $\eta$-derivatives of the normalization factor $\mathcal{U}_{\kappa}$ characterize the difference between the double-escort $\kappa$-expectation and the $\kappa$-escort expectation.

By using the double-escort pdf, we obtain:

$$
\partial_{i} P(x ; \boldsymbol{\theta})=\frac{1}{\mathcal{U}_{\kappa}}\left\{\tilde{\mathcal{U}}_{\kappa} \tilde{P}(x ; \boldsymbol{\theta})\left(f_{i}(x)-\mathbb{E}_{P}\left[f_{i}\right]\right)-P(x ; \boldsymbol{\theta}) \partial_{i} \mathcal{U}_{\kappa}\right\}
$$

Substituting Relation (101) into this and after some calculations, we finally obtain that:

$$
g_{i j}^{(\kappa-\mathrm{esc})}=\partial_{i} \mathbb{E}_{P}\left[f_{j}\right]=\int d x \partial_{i} P(x ; \boldsymbol{\theta}) f_{j}(x)=\frac{\tilde{\mathcal{U}}_{\kappa}}{\mathcal{U}_{\kappa}} \tilde{\mathbb{E}}_{\tilde{P}}\left[\left(f_{i}-\mathbb{E}_{P}\left[f_{i}\right]\right)\left(f_{j}-\mathbb{E}_{P}\left[f_{j}\right]\right)\right],
$$

which is the $\kappa$-generalization of the fluctuation-response relation associated with the double-escort $\kappa$-expectations. To the best of our knowledge, this is the first report to show Relation (103) in the literature.

Next, similar to the representation $\ell_{\theta}^{(\kappa)}$, we consider the quantity:

$$
\mathrm{f}_{\theta}^{(\kappa)} \equiv \lambda u_{\kappa}\left(\frac{p(x ; \boldsymbol{\theta})}{\alpha}\right)
$$

as the co-representation of $\ell_{\theta}^{(\kappa)}$. From Relation (58), we see that:

$$
\mathrm{f}_{\theta}^{(\kappa)}=\sqrt{\lambda^{2}+\kappa^{2}\left(\sum_{m} \theta^{m} f_{m}(x)-\gamma(\boldsymbol{\theta})\right)^{2}} .
$$

We think that this new quantity represents a certain kind of fluctuation characterized by the deformed parameter $\kappa$. The difference between the expectation of $\ell_{\theta}^{(\kappa)}$ and that of $\mathrm{t}_{\theta}^{(\kappa)}$ is:

$$
\begin{aligned}
\mathrm{E}_{p}\left[\ell_{\theta}^{(\kappa)}\right]-\mathrm{E}_{p}\left[\mathrm{f}_{\theta}^{(\kappa)}\right] & =\mathrm{E}_{p}\left[\sum_{m} \theta^{m} f_{m}(x)-\gamma(\boldsymbol{\theta})\right]-\mathrm{E}_{p}\left[u_{\kappa}(p(x ; \boldsymbol{\theta}))+\kappa^{2} \ln _{\kappa} p(x ; \boldsymbol{\theta})\right] \\
& =\boldsymbol{\theta} \cdot \eta-\gamma(\boldsymbol{\theta})-\mathcal{I}_{\kappa}-\kappa^{2} \Psi_{\kappa}^{\star}(\boldsymbol{\eta})=\boldsymbol{\theta} \cdot \eta-\Psi_{\kappa}(\boldsymbol{\theta})-\kappa^{2} \Psi_{\kappa}^{\star}(\boldsymbol{\eta}) \\
& =\left(1-\kappa^{2}\right) \Psi_{\kappa}^{\star}(\boldsymbol{\eta}) .
\end{aligned}
$$

Note that this relation is non-trivial unless $\kappa=0$ and in the limit of $\kappa \rightarrow 0$ Relation (106) reduces to the definition $\mathrm{E}_{p}[\ln p(x ; \boldsymbol{\theta})]=\Psi^{\star}(\boldsymbol{\eta})$.

Taking the $\kappa$-escort expectation of (105), we see that:

$$
\mathbb{E}_{P}\left[\mathfrak{l}_{\theta}^{(\kappa)}\right]=\frac{1}{\mathcal{U}_{\kappa}} \int d x \frac{p(x ; \boldsymbol{\theta})}{\lambda u_{\kappa}\left(\frac{p(x ; \boldsymbol{\theta})}{\alpha}\right)} \lambda u_{\kappa}\left(\frac{p(x ; \boldsymbol{\theta})}{\alpha}\right)=\frac{1}{\mathcal{U}_{\kappa}} \int d x p(x ; \boldsymbol{\theta})=\frac{1}{\mathcal{U}_{\kappa}},
$$

which states that the normalization factor $\mathcal{U}_{\kappa}$ of the $\kappa$-escort pdf characterizes the expectation of the above fluctuations. Next, taking derivative of $\mathrm{f}_{\theta}^{(\kappa)}$ with respect to $\eta_{i}$, we have:

$$
\partial^{i} \mathfrak{Y}_{\theta}^{(\kappa)}=\kappa^{2} \frac{\left(\sum_{m} \theta^{m} f_{m}(x)-\gamma(\boldsymbol{\theta})\right) \partial^{i}\left(\sum_{m} \theta^{m} f_{m}(x)-\gamma(\boldsymbol{\theta})\right)}{\sqrt{\lambda^{2}+\kappa^{2}\left(\sum_{m} \theta^{m} f_{m}(x)-\gamma(\boldsymbol{\theta})\right)^{2}}}=\kappa^{2} \frac{\lambda \ln _{\kappa}\left(\frac{p(x)}{\alpha}\right)}{\lambda u_{\kappa}\left(\frac{p(x)}{\alpha}\right)} \partial^{i} \ell_{\theta}^{(\kappa)} .
$$


Then, the expectation of the tangent vector $\partial^{i} \mathrm{f}_{\theta}^{(\kappa)}$ becomes:

$$
\begin{aligned}
\mathrm{E}_{p}\left[\partial^{i} \mathfrak{1}_{\theta}^{(\kappa)}\right] & =\int d x p(x) \partial^{i} \lambda u_{\kappa}\left(\frac{p(x)}{\alpha}\right)=\int d x p(x) \frac{\kappa^{2} \lambda \ln _{\kappa}\left(\frac{p(x)}{\alpha}\right)}{p(x)} \partial^{i} p(x) \\
& =\kappa^{2} \int d x \lambda \ln _{\kappa}\left(\frac{p(x)}{\alpha}\right) \partial^{i} p(x)=\kappa^{2} \partial^{i} \int d x p(x) \ln _{\kappa} p(x) \\
& =\kappa^{2} \partial^{i} \Psi_{\kappa}^{\star}(\boldsymbol{\eta})=\kappa^{2} \theta^{i},
\end{aligned}
$$

which is the corresponding relation to (82).

Finally, we derive the canonical divergences for the two dualistic Hessian structures. For the $\kappa$-deformed exponential pdf $p(x ; \boldsymbol{\theta})$ and an arbitrary pdf $r(x)$, we have:

$$
\begin{aligned}
\int d x r(x) \ln _{\kappa} p(x ; \boldsymbol{\theta}) & +\int d x r(x) u_{\kappa}(p(x ; \boldsymbol{\theta}))=\int d x r(x) \lambda \ln _{\kappa}\left(\frac{p(x ; \boldsymbol{\theta})}{\alpha}\right) \\
& =\int d x r(x)\left[\sum_{m}\left(\theta_{p}\right)^{m} f_{m}(x)-\gamma\left(\boldsymbol{\theta}_{p}\right)\right]=\boldsymbol{\theta}_{p} \cdot \boldsymbol{\eta}_{r}-\gamma\left(\boldsymbol{\theta}_{p}\right) .
\end{aligned}
$$

Then, the canonical divergence [13] associated with the potential functions $\Psi_{\kappa}(\boldsymbol{\theta})$ and $\Psi_{\kappa}^{\star}(\boldsymbol{\eta})$ becomes:

$$
\begin{aligned}
D^{(\kappa)}(\boldsymbol{p}, \boldsymbol{r}) & =\Psi_{\kappa}^{\star}\left(\boldsymbol{\eta}_{r}\right)-\left(\boldsymbol{\theta}_{p} \cdot \boldsymbol{\eta}_{r}-\Psi_{\kappa}\left(\boldsymbol{\theta}_{p}\right)\right) \\
& =\int d x r(x)\left[\ln _{\kappa} r(x)-\ln _{\kappa} p(x ; \boldsymbol{\theta})-u_{\kappa}(p(x ; \boldsymbol{\theta}))\right]+\mathcal{I}_{\kappa}\left(\boldsymbol{\theta}_{p}\right) \\
& =\int d x r(x)\left[\ln _{\kappa} r(x)-\ln _{\kappa} p(x ; \boldsymbol{\theta})\right]-\int d x[r(x)-p(x ; \boldsymbol{\theta})] u_{\kappa}(p(x ; \boldsymbol{\theta})),
\end{aligned}
$$

which reduces to the KL divergence (39) in the limit of $\kappa \rightarrow 0$.

Similarly, for the $\kappa$-deformed exponential pdf $p(x ; \boldsymbol{\theta})$ and an arbitrary pdf $r(x)$, we have:

$$
\mathbb{E}_{R}\left[\ell_{\theta_{p}}^{(\kappa)}\right]=\mathbb{E}_{R}\left[\sum_{m}\left(\theta_{p}\right)^{m} f_{m}(x)-\gamma\left(\boldsymbol{\theta}_{p}\right)\right]=\boldsymbol{\theta}_{p} \cdot \boldsymbol{\eta}_{r}^{\mathrm{esc}}-\gamma\left(\boldsymbol{\theta}_{p}\right),
$$

where $\mathbb{E}_{R}[]$ denotes the $\kappa$-escort expectation with respect to $r(x)$. Then, the canonical divergence associated with the potential functions $\Psi_{\kappa}^{\mathrm{esc}}(\boldsymbol{\theta})$ and $\left(\Psi^{\mathrm{esc}}\right)_{\kappa}^{\star}(\boldsymbol{\eta})$ becomes:

$$
\begin{aligned}
D^{(\mathrm{esc}-\kappa)}(\boldsymbol{p}, \boldsymbol{r}) & =\left(\Psi_{\kappa}^{\mathrm{esc}}\right)^{\star}\left(\boldsymbol{\eta}_{r}\right)-\left(\boldsymbol{\theta}_{p} \cdot \boldsymbol{\eta}_{r}^{\mathrm{esc}}-\Psi_{\kappa}^{\mathrm{esc}}\left(\boldsymbol{\theta}_{p}\right)\right)=\mathbb{E}_{P}\left[\ell_{\theta_{r}}^{(\kappa)}\right]-1-\left(\boldsymbol{\theta}_{p} \cdot \boldsymbol{\eta}_{r}^{\mathrm{esc}}-\gamma\left(\boldsymbol{\theta}_{p}\right)-1\right) \\
& =\mathbb{E}_{P}\left[\ell_{\theta_{r}}^{(\kappa)}\right]-\mathbb{E}_{R}\left[\ell_{\theta_{p}}^{(\kappa)}\right] \\
& =\mathbb{E}_{P}\left[\ln _{\kappa} r(x)+u_{\kappa}(r(x))\right]-\mathbb{E}_{R}\left[\ln _{\kappa} p(x ; \boldsymbol{\theta})+u_{\kappa}(p(x ; \boldsymbol{\theta}))\right],
\end{aligned}
$$

which also reduces to the KL divergence (39) in the limit of $\kappa \rightarrow 0$.

\section{Conclusions}

We have studied the dualistic Hessian geometries among the thermodynamic potentials in the $\kappa$-thermostatistics. Since a deformed exponential pdf naturally induces the escort pdf [8] in general, the $\kappa$-deformed exponential pdf also induces the $\kappa$-escort pdf. Consequently, it is important to take into account both kinds of expectations: one is the $\kappa$-escort expectation, and the other is the standard expectation. The Legendre relations among the thermodynamic potentials concerning both expectations 
are explored, and we have found a remarkable feature that for the affine-coordinate $\boldsymbol{\theta}$, there exist the two different kinds ( $\boldsymbol{\eta}$ and $\boldsymbol{\eta}^{\text {esc }}$ ) of the dual affine-coordinates. The two different $\kappa$-deformed metrics $g^{(\kappa)}$ and $g^{(\kappa-e s c)}$ are related to the thermodynamic fluctuations (95) and (103), respectively. Particularly, in order to establish the new $\kappa$-generalization (103) of the fluctuation-response relation, we introduced the double-escort $\kappa$-expectation given in (100). We believe that these $\kappa$-generalizations of the fluctuation-response relation play important roles in a future study of a non-equilibrium thermodynamic system described in the $\kappa$-thermostatistics.

Further studies are necessary to understand the roles of the different kinds of the dual affine-coordinates in thermodynamic geometries concerning the deformed functions. In addition, since the independence of an exponential pdf plays a fundamental role in the equilibrium statistical physics and thermodynamics, it is interesting to further study the generalization of the independence on a deformed pdf [20] based on the results obtained in this study.

\section{Acknowledgments}

The first named author is partially supported by the Japan Society for the Promotion of Science (JSPS) Grants-in-Aid for Scientific Research (KAKENHI) Grant Number 25400188. The second named author is partially supported by the JSPS Grants-in-Aid for Scientific Research (KAKENHI) Grant Number 26108003 and $15 \mathrm{~K} 04842$.

\section{Author Contributions}

Tatsuaki Wada designed the main part of the research with the help of the rest of authors and mainly wrote the manuscript. Hiroshi Matsuzoe and Antonio M. Scarfone commented on the manuscript at all stages. All authors equally performed the research and discussed the results. All authors have read and approved the final manuscript.

\section{Conflicts of Interest}

The authors declare no conflict of interest.

\section{References}

1. Gibbs, J.W. A Method of Geometrical Representation of the Thermodynamic Properties of Substances by Means of Surfaces. Trans. Conn. Acad. 1873, II, 382-404.

2. Pogliani, L.; Berberan-Santos, M.N. Constantin Carathéodory and the axiomatic thermodynamics. J. Math. Chem. 2000, 28, 313-324.

3. Ruppeiner, G. Riemannian geometry in thermodynamic fluctuation theory. Rev. Mod. Phys. 1995, 67, 605-659.

4. Weinhold, F. Metric geometry of equilibrium thermodynamics, I-IV. J. Chem. Phys. 1975, 63, 2479-2501.

5. Weinhold, F. Metric geometry of equilibrium thermodynamics, V. J. Chem. Phys. 1976, 65, 559-564. 
6. Amari, S.; Nagaoka, H. Methods of Information Geometry; American Mathematical Society: Providence, RI, USA, 2000.

7. Tsallis, C. Introduction to Nonextensive Statistical Mechanics: Approaching a Complex World; Springer: New York, NY, USA, 2009.

8. Naudts, J. Generalized Thermostatistics; Springer: Berlin, Germany, 2011.

9. Kaniadakis, G.; Scarfone, A.M. A new one-parameter deformation of the exponential function. Physica A 2002, 305, 69-75.

10. Kaniadakis, G. Statistical mechanics in the context of special relativity. Phys. Rev. E 2002, 66, 056125.

11. Kaniadakis, G. Statistical mechanics in the context of special relativity II. Phys. Rev. E 2005 72, 036108.

12. Kaniadakis, G. Maximum entropy principle and power-law tailed distributions. Eur. J. Phys. B 2009, 70, 3-13.

13. Scarfone, A.M.; Wada, T. Legendre structure of $\kappa$-thermostatistics revisited in the framework of information geometry. J. Phys. A Math. Theor. 2014, 47, 275002.

14. Kaniadakis, G. Theoretical foundations and mathematical formalism of the power-law tailed statistical distributions. Entropy 2015, 15, 3913-4010.

15. Callen, H.B. Thermodynamics; Wiley: New York, NY, USA, 1960.

16. Wada, T.; Scarfone, A.M. Information geometry on the $\kappa$-thermostatistics. Entropy 2015, 17, 1204-1217.

17. Matsuzoe, H.; Henmi, M. Hessian structures and divergence functions on deformed exponential families. In Geometric Theory of Information, Signals and Communication Technology; Nielsen, F., Ed.; Springer: Berlin, Germany, 2014; pp. 57-80.

18. Brillouin, L. Negentropy Principle of Information. J. Appl. Phys. 1953, 24, 1152-1163.

19. Scarfone, A.M.; Wada, T. Canonical partition function for anomalous systems described by the $\kappa$-entropy. Prof. Theor. Phys. Suppl. 2006, 162, 45-52.

20. Matsuzoe, H.; Wada, T. Deformed algebras and generalizations of independence on deformed exponential families. Entropy 2015, 17, 5729-5751.

(C) 2015 by the authors; licensee MDPI, Basel, Switzerland. This article is an open access article distributed under the terms and conditions of the Creative Commons Attribution license (http://creativecommons.org/licenses/by/4.0/). 